\title{
Origines, thèmes et enjeux de la CNUED
}

Andràs November, Jacques Grinevald, Pierre Portas et Christophe Dunand

\section{OpenEdition}

\section{Journals}

Édition électronique

URL : http://journals.openedition.org/aspd/1561

DOI : 10.4000/aspd.1561

ISSN : 1663-9669

\section{Éditeur}

Institut de hautes études internationales et du développement

\section{Édition imprimée}

Date de publication : 1 janvier 1992

Pagination : 159-175

ISSN : 1660-5934

\section{Référence électronique}

Andràs November, Jacques Grinevald, Pierre Portas et Christophe Dunand, « Origines, thèmes et enjeux de la CNUED », Annuaire suisse de politique de développement [En ligne], 11 | 1992, mis en ligne le 18 mai 2013, consulté le 08 septembre 2020. URL : http://journals.openedition.org/aspd/1561 ; DOI : https://doi.org/10.4000/aspd. 1561 


\title{
Origines, thèmes et enjeux de la CNUED
}

\author{
Andràs November, Jacques Grinevald, \\ Pierre Portas et Christophe Dunand
}

\section{Les antécédents de la Conférence de Rio sur l'environnement et le dévelop- pement (CNUED)}

Pour appréhender correctement les thèmes et les enjeux de la grande conférence de Rio de Janeiro sur l'environnement et le développement, il est nécessaire de bien situer cet événement dans son contexte historique. $\mathrm{Si}$, pour l'opinion publique, c'est la conférence de Stockholm sur "l'environnement humain", du 5 au 16 juin 1972, qui marque le début de la prise de conscience de la problématique mondiale de l'environnement, l'origine du débat sur l'environnement et le développement remonte plus précisément à la réunion préparatoire de Founex (dans les environs de Genève), les 4 ou 12 juin 1971. Cette réunion, avec des représentants de pays du Tiers Monde, et le rapport qu'elle rédigea soulignèrent le fait que les problèmes environnementaux dans les pays du Tiers Monde faisaient partie de la révision et de l'élargissement du concept de développement lui-même (1).

Mais pour comprendre le cheminement de cette idée, remontons encore à l'année 1968, en rappelant la "conférence intergouvernementale d'experts sur les bases scientifiques de l'utilisation rationnelle et de la conservation des ressources de la biosphère" organisée à Paris, les 4-13 septembre 1968, par l'Unesco (2), et où nacquit l'idée même de la conférence de Stockholm, et la première conférence internationale sur "les aspects écologiques du développement international" organisé les 8-11 décembre 1968 à Airlie House, en Virginie, par la Conservation Foundation (Washington) et la Washington University de St. Louis(3). Suite à cette conférence, la Conservation Foundation et l'Union Internationale pour la Conservation de la Nature et de ses Ressources (UICN), à Morges (Suisse), publia un manuel intitulé Ecological Principles for Economic Development. (4) 
Vers 1970, année charnière de "la révolution de l'environnement" (Max Nicholson), de la prise de conscience de la "crise écologique", les réalités de la arrivent brutalement sur le devant de la scène publique: tout le monde se met à parler de la Biosphère pollution, des ressources naturelles, de la dégradation de la nature; les termes mêmes d'écologie et d'environnement entrent dans le vocabulaire usuel et deviennent des enjeux politiques, juridiques et culturels (5).

Les aspects écologiques du développement deviennent soudain une nouvelle dimension d'une situation internationale déjà fort complexe. Les recherches scientifiques sur l'environnement, à tous les niveaux, du plus local au plus global, se développent aussi rapidement, mais l'écologie scientifique reste souvent peu favorisée dans les politiques de la science, de la technologie et de l'éducation. Les sciences sociales, fondées sur le postulat de la séparation culture/nature réagissent avec retard et embarras. La science économique se voit de plus en plus questionnée sur sa capacité théorique et méthodologique à réconcilier ses analyses abstraites avec l'économie réelle - thermodynamique - de la nature dans laquelle s'insère pourtant le processus économique, comme l'a amplement démontré Georgescu-Roegen (6).

Depuis la Conférence de l'Unesco sur la Biosphère de 1968, le point de vue des écologistes a fait son chemin. Mais on peut dire sans risque de se tromper que jusqu'à la fin des années 80 , peu de responsables des milieux politiques et économiques prirent au sérieux le discours apparemment catastrophiste des écologistes. La publication retentissante, en mars 1972, du "rapport Meadows" sur Les limites à la croissance, le fameux premier rapport au Club de Rome, fut accueilli par un tollé général, notamment dans les rangs des économistes, qu'ils soient de tendance libérale ou socialiste. Au niveau scientifique, le débat se poursuivit en dehors de l'establishment de la science économique par quelques dissidents en quête d'une "économie écologique".

Dès la fin des années 60 les bases du débat sur l'environnement et le développement étaient posées. Mais la situation politique internationale était différente et même si les multiples problèmes écologiques du développement étaient déjà très sérieux, on n'en mesurait pas encore les plus graves effets à l'échelle globale de la Biosphère.

A vrai dire, au début des années 70 , on voyait la crise de l'environnement comme essentiellement une affaire de pays industrialisés. Le monde communiste, qui n'était guère représenté à Stockholm, déclarait même qu'il s'agissait d'un problème typique du capitalisme libéral! Les dirigeants des pays dits sousdéveloppés, fascinés par "la tentation de l'Occident" (Malraux), cherchaient d'abord à rattraper leur retard technologique et économique: les préoccupations écologiques, luxe de nantis pense-t-on alors, viendraient ensuite, avec l'abondance. Aujourd'hui, il est évident pour tout le monde que l'écologie du développement économique ne se partage pas aussi sommairement: la crise écologique concerne l'Est comme l'Ouest, le Sud comme le Nord, naturellement selon des modalités spécifiques. La géographie de l'industrialisation et de la militarisation a changé, elle est devenue planétaire. Les impacts écologiques des activités des 
sociétés de la Terre, activités tant industrielles, militaires, agricoles que sociales, ne se produisent plus seulement au niveau local ou régional, mais également au niveau global. Le problème de la dérive anthropique de l'effet de serre réchauffement du globe dont les conséquences climatiques, biologiques, économiques et sociales font actuellement l'objet de nombreuses études et discussions internationales - en est un exemple préocuppant (7).

Dès 1985, la réunion OMM-PNUE-CIUS des experts du climat à Villach aboutit à un consensus scientifique sans précédent à propos de la gravité du problème de l'augmentation accélérée de la concentration du gaz carbonique et d'autres gaz à effet de serre dans l'atmosphère. La question, au plan scientifique, n'était d'ailleurs pas nouvelle. II fallut cependant attendre l'été 1988 pour que la question prenne une tournure nettement plus politique, comme le souligna la conférence mondiale sur "l'atmosphère en évolution: implications pour la sécurité du globe", tenue à Toronto, en juin 1988 et qui fut rapidement suivie d'une quantité impressionnante d'autres réunions internationales et de déclarations du même genre (8).

La principale illustration des changements récents dans la prise en compte de la dimension écologique du développement économique est le rapport de la Commission mondiale sur l'environnement et le développement, le rapport Bruntdland, publié au printemps 1987. Assez semblable mais plus alarmiste encore que le rapport Only One Earth de 1972 (9), plus ambitieux que la Stratégie Mondiale de la Conservation publiée par le WWF, I'UICN et le PNUE en 1980, Our Common Future (traduit par "Notre avenir à tous") a été un tournant majeur dans la prise de conscience internationale de la problématique environnementdéveloppement. Dressant un bilan assez impressionnant, il insiste notamment sur la nécessité de promouvoir un "développement durable", de procéder à une "réorientation des attitudes et des priorités", de s'attaquer aux problèmes fondamentaux comme la militarisation, l'explosion démographique et la pauvreté (10).

La panution de ce rapport a fait avancer d'un grand pas la prise en compte de la dimension écologique du développement économique. Cependant, alors que ce document insiste sur l'importance de l'identification des racines des problèmes, il est présupposé que la croissance économique est un élément déterminant pour lever les contraintes qui mènent à la dégradation de l'environnement.

En novembre 1988, l'Organisation mondiale météorologique (OMM) et le Programme des Nations Unies pour l'environnement (PNUE) instituèrent conjointement l'Intergovernmental Panel on Climate Change (IPCC), dont le rapport, assez alarmiste, fut présenté en 1990 à la deuxième Conférence mondiale sur le climat, à Genève (11). Le travail remarquable de l'IPCC présente le consensus de la communauté scientifique internationale sur la réalité des menaces climatiques et écologiques liées à l'accroissement de l'effet de serre dont est responsable, de manière très inégale, l'ensemble de l'humanité en développement, mais plus particulièrement la croissance de la civilisation industrielle.

Le contexte politique subit aussi des transformations profondes, particulièrement dans le domaine stratégique des relations Est-Ouest, mais les enjeux de la dégradation de l'environnement et du destin de la Biosphère sont encore loin de faire l'unanimité, tant au Nord - qui cherche à minimiser ses responsabilités - 
qu'au Sud, où on rejette volontiers toute la responsabilité dans le camp des pays les plus industrialisés. Certes, les problèmes d'environnement ne sont plus systématiquement rélégués à l'après-sous-développement, mais trop souvent le manque de moyens économiques a raison des initiatives politiques.

Comme la Banque mondiale depuis peu, les grandes compagnies industrielles sont aussi en train de se "mettre au vert", tout au moins dans le discours officiel et les médias. Cependant, vingt ans après Stockholm, force est de constater que les questions de fonds ne sont toujours pas résolues, à peine sont-elles reconnues, et que l'ampleur des périls à venir ne fait que croître.

La communauté scientifique internationale est à présent quasiment unanime pour dire que la situation est nettement plus préoccupante qu'il y a vingt ans. Les recherches scientifiques récentes sur l'évolution de la Biosphère, notamment sur ses cycles biogéochimiques et son climat global, mettent en évidence l'ampleur véritablement planétaire de la crise écologique du développement de l'espèce humaine. C'est toute la Biosphère - et le concept Gaïa, dont on parle de plus en plus, souligne bien que nous en faisons partie au même titre que des millions d'autres espèces vivantes - qui est aujourd'hui en péril (12). La gravité de cette crise sans précédent dans toute l'histoire "humaine" de la Biosphère souligne l'ampleur du défi de la conférence de Rio 92.

\section{Vers le "Sommet Planète Terre» : la préparation de la Conférence de Rio}

C'est sous la dénomination symbolique "Sommet Planète Terre" que se tiendra la Conférence des Nations Unies sur l'environnement et le développement (CNUED) à Rio de Janeiro, au Brésil, du 1er au 12 juin 1992. Elle a été convoquée par une décision du 22 décembre 1989 (Résolution 44/228) de l'Assemblée générale des Nations Unies pour commémorer le 20ème anniversaire de la Conférence de Stockholm. Cette décision marque aussi concrètement la volonté des Nations Unies de s'engager dans la voie ouverte par les conclusions de la Commission mondiale sur l'environnement et le développemnent (autrement dit du rapport Brundtland).

La Conférence de Rio - qui sera une des plus importante dans les annales des Nations Unies et probablement l'événements de l'année 1992 - réunira environ vingt mille participants de plus de cent pays du monde entier. Le point culminant de la CNUED sera la célébration de la Journée mondiale de l'environnement, le 5 juin, en présence des chefs d'Etat et de gouvernements.

En même temps ce sommet intergouvernemental sera entouré par de nombreuses manifestations parallèles, dont une exposition sur la technologie des équipements de protection de l'environnement et un cycle d'études sur la science et la technologie. Enfin, un symposium des "secteurs indépendants", qui réunira plus de deux cents organisations non gouvernementales et associations provenant de plus de soixante pays, est annoncé comme un des événements marquants; il se déroulera en marge de la Conférence (13). 
L'Assemblée générale de l'ONU a confié l'organisation de la Conférence à un Secrétariat, basé à Genève et dirigé par M. Maurice Strong (ancien membre de la Commission Brundtland et déjà à la tête du Secrétariat de Stockholm 72), qui a mis en place un Comité préparatoire. En quatre sessions à Nairobi, à Genève (à deux reprises) et à New York le Comité préparatoire doit baliser le terrain, établir l'ordre du jour et élaborer les documents qui seront soumis à la Conférence de Rio.

Le secrétariat de la CNUED a initié un processus de consultation des organisations gouvemementales et non gouvernementales qui s'intéressent aux problèmes de l'environnement et du développement. Le but est de solliciter les avis d'un large éventail d'institutions allant des petits groupes de citoyens jusqu'aux hautes instances des Nations Unies, en passant par les diverses organisations spécialisées, les organismes régionaux, les institutions de recherche, la communauté scientifique et universitaire et le monde associatif et militant en faveur de l'environnement. Selon le vœux des organisateurs, pendant la période préparatoire de la conférence, toutes les forces actives dans ces domaines devraient converger vers la conférence.

Les principaux thèmes de la conférence sont les suivants:

a) Problèmes de développement en relation avec l'environnement (questions intersectorielles):

- Relation entre la pauvreté et la dégradation de l'environnement;

- Relation entre les pressions démographiques, les modes de consommation, l'absence de croissance et la dégradation de l'environnement;

- Economie internationale et environnement;

- Comptablitité intégrée économie-environnement;

- Transferts de technologie;

- Nouvelles ressources financières pour les pays du Tiers Monde;

- Hygiène de l'environnement et le développement;

- Education, formation et sensibilisation du public à un développement durable.

b) Problèmes écologiques globaux:

- Protection de l'atmosphère (changements climatiques, appauvrissement de la couche d'ozone, pollution atmosphérique transfrontière);

- Protection des sols (déboisement, pertes en terres, désertification et séchéresse);

- Conservation et développement des forêts;

- Présenvation de la diversité biologique;

- Gestion écologiquement rationnelle des biotechnologies;

- Aspects mondiaux de la pollution marine;

- Protection des ressources en eau douce et utilisation rationnelle des ressources en eau dans l'agriculture;

- Gestion écologiquement rationnelle des déchets toxiques, particulièrement des déchets dangereux et des produits chimiques toxiques. 
Pour approfondir les différents thèmes et maîtriser le grand nombre de documents transmis au Secrétariat de la CNUED, le Comité préparatoire a créé trois groupes de travail qui jouent un rôle important dans l'élaboration des documents et dans la formulation des propositions qui seront soumises à la Conférence de Rio.

Enfin, pour améliorer l'information de nombreuses organisations impliquées dans la préparation de la CNUED, un système d'information électronique a été instauré, dont l'accès est libre à tous ceux qui souhaitent avoir accès à l'un ou l'autre des documents préparés pour la conférence (14). Le déroulement du travail préparatoire et les liaisons avec d'autres instances des Nations Unies, avec les organisations non gouvernementales, avec les instituts de recherche scientifiques et universitaires, peut être illustré par le schéma ci-dessous.

\section{Que peut-on attendre de la CNUED?}

Les objectifs du "Sommet Planète Terre" sont très ambitieux, d'une ampleur et d'une complexité immenses. II s'agit de trouver des réponses aux problèmes planétaires de l'environnement et du développement. "La nécessité d'intégrer l'environnement et le développement à tous les niveaux de l'élaboration des politiques et la prise de décisions, ainsi que leur incorporation dans les programmes et dans les projets, est au cœur des préoccupations de la CNUED." (15).

Pour ce faire, la Conférence devrait établir les fondements d'une nouvelle conception de la coopération internationale qui permettra un développement durable à long terme des sociétés humaines. Comme l'écrit M. Maurice Strong, Secrétaire général de la CNUED: «Si nos efforts sont couronnés de succès, nous pouvons espérer produire une toute nouvelle vision, dont les proportions risquent de surprendre même les plus avertis d'entre nous. Partout, le comportement des gouvernements, mais aussi des individus, en sera modifié." (16).

Plus concrètement, il est prévu que pendant la CNUED, les chefs d'Etat et de gouvernements signeront plusieurs accords internationaux, notamment la convention sur le climat qui englobe la protection de la couche d'ozone, la réduction de la teneur de l'atmosphère en $\mathrm{CO} 2$ et autres gaz à effet de serre, la protection des forêts tropicales, la convention sur la sylviculture, l'accord sur la sauvegarde des eaux, ou encore la convention sur la protection de la diversité biologique.

En même temps, la CNUED devrait adopter une déclaration sur les principes fondamentaux concernant l'interdépendance entre environnement et développement, sous la forme d'une "Charte de la Terre". Ce document énoncera "les règles de conduite des peuples, les uns par rapport aux autres et vis-à-vis de l'environnement" (17). Cette déclaration solennelle constituera une sorte de "devoir moral de protéger l'environnement" (selon le représentant du Saint Siège), un premier pas vers une éthique de l'environnement, mais ne revêtira en fait aucune obligation juridique pour les signataires.

La Déclaration de Rio sera complétée par un programme d'activité dénommé "Agenda 21 ". Le Comité préparatoire de la CNUED attribue une importance primordiale à ce document: il doit établir l'inventaire des problèmes liés à l'environ- 
PREPARATION DU "SOMMET PLANETE TERRE"

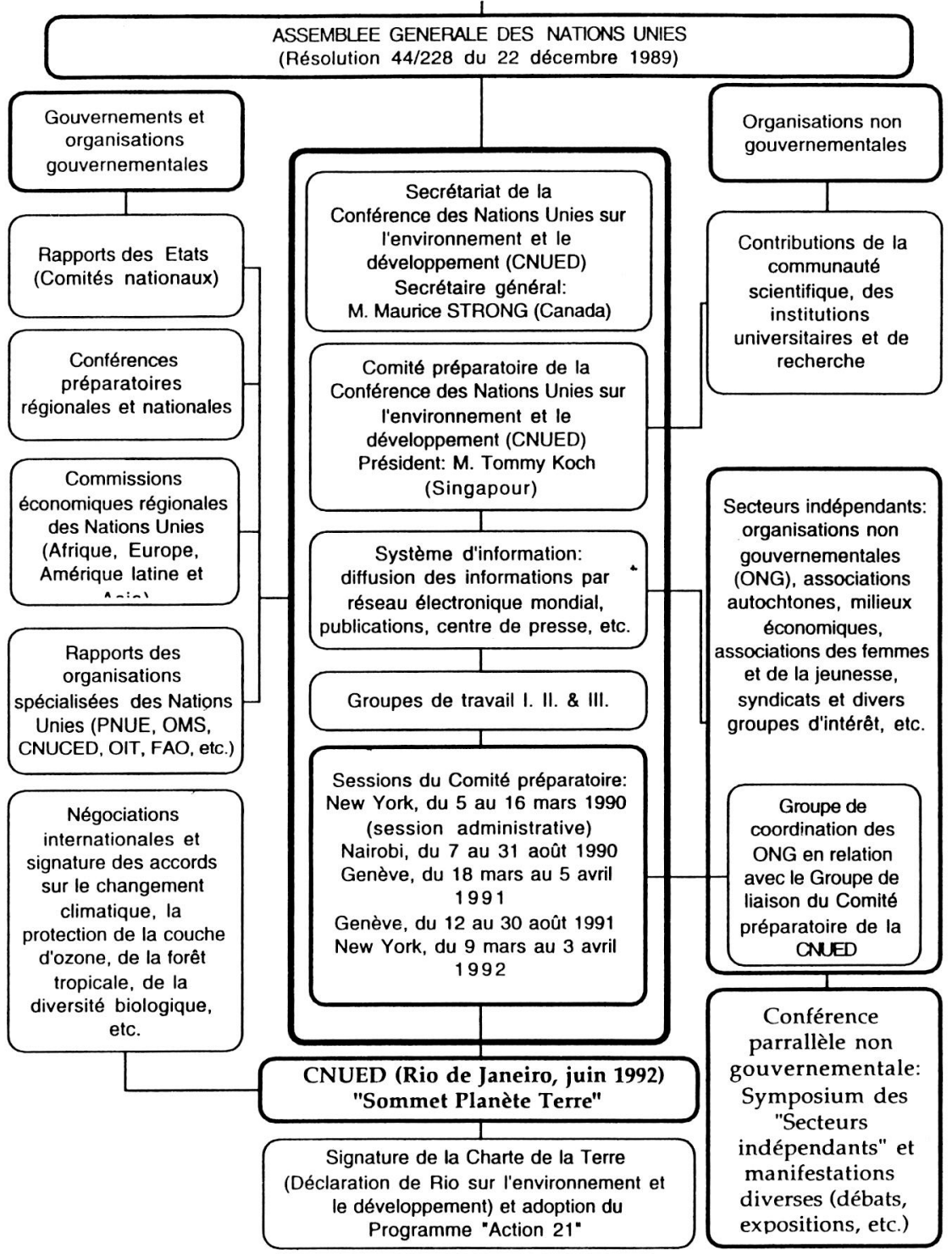


nement et au développement, étudier leurs interrelations, fixer les objectifs et tracer les mesures concrètes que les Etats devraient entreprendre jusqu'à la fin du siècle prochain, d'où son nom: Action 21 ou Agenda 21. Le programme contenu dans ce document servira de cadre de référence pour les gouvernements, les organisations internationales, les milieux économiques et d'autres acteurs, telles que les ONG, pour mettre en œuvre la politique du développement - socioécologiquement - durable.

En outre, la Conférence doit formuler les recommendations concernant les ressources financières, les modalités de transfert de technologies non polluantes et le renforcement des institutions internationales qui prendront en charge l'exécution des tâches qui découleront des décisions de la CNUED. Ces objectifs sont ambitieux mais ils pourraient l'être encore plus s'il était prévu de se pencher sur les causes profondes des problèmes d'environnement et de développement actuels. La perspective générale est proche de celle du rapport Brundtland qui a une grande influence sur le travail de préparation de la conférence.

\section{Environnement versus développement}

La relation entre environnement et développement est donc au centre des débats de la CNUED. Même s'il est vrai que "le développement et l'environnement ne forment que les deux faces de la même médaille", selon l'expression de Mustafa Tolba, Directeur du Programme des Nations Unies pour l'environnement (PNUE), les deux concepts possèdent des racines différentes et ne peuvent être facilement intégrés. On ne peut pas simplement les juxtaposer: ils sont à la fois convergents et divergents, complémentaires et antagonistes. Par ailleurs ils agissent différemment à court terme et à long terme, dans une relation dialectique.

Dans l'esprit de nombreux acteurs et observateurs du Tiers Monde, la notion de développement est toujours étroitement liée à la croissance économique. Le renforcement de l'infrastructure économique constitue une préoccupation majeure et la prise en compte des limites écologiques du développement passe au second plan. Dans cette perspective, les mesures de protection de l'environnement doivent s'appuyer sur les moyens créés par le processus du développement économique lui-même.

"Notre démarche, dit le rapport Brundtland, n'est pas de prévoir une dégradation constante de l'environnement, une progression de la pauvreté et des difficultés dans un monde de plus en plus pollué où les ressources seraient de moins en moins nombreuses. Nous envisageons plutôt la possibilité d'une nouvelle ère de croissance économique, s'appuyant sur des politiques qui protégeraient, voire même mettraient en valeur les ressources. Nous estimons que cette croissance est absolument indispensable pour soulager la misère qui ne fait que s'intensifier dans une bonne part du monde en développement. Mais l'espoir que la Commission place en l'avenir est conditionné par la prise immédiate de mesures politiques décisives pour commencer à gérer les ressources de l'environnement de manière à assurer un progrès durable et garantir la survie de l'humanité". 
La Commission Brundtland, qui plaide en faveur de la croissance économique, estime que seul le "développement durable" du point de vue écologique permet de lever l'hypothèque qui pèse sur la croissance destructrice de l'environnement. Ainsi, le développement durable - notion aussi floue qu'indispensable - est définit comme le processus qui “répond aux besoins du présent sans compromettre la capacité des générations futures de répondre à leurs propres besoins". II s'agit d'une définition - parmi bien d'autres - du "développement durable" qui met l'accent sur l'interrelation entre la solidarité synchronique avec les pays du Tiers Monde et la solidarité diachronique avec les générations futures qui nous obligent à sauvegarder la Biosphère.

II faut se garder de croire que le "développement durable" est un concept pour l'usage exclusif du Tiers Monde. II met également en cause l'attitude des pays industrialisés du Nord, à l'Est comme à l'Ouest. Sur-développement du Nord et sous-développement au Sud conduisent l'un et l'autre au “mal-développement" et à la destruction de l'environnement. Ce fait est d'ailleurs admis aujourd'hui par le Conseil des industriels qui soutiennent le développement durable (Business Council for Sustainable Development) formé en marge de la Conférence de Rio sous l'impulsion de M. Stephan Schmidheiny. (18)

Le développement durable implique plus que des modifications cosmétiques de l'économie mondiale et des pratiques dominantes de gestion des ressources et de l'environnement qui en découlent. La logique économique du marché ne permet pas une gestion à long terme des ressources et de la pollution car elle néglige l'ensemble des processus naturels et des produits dépourvus de valeur marchande, qui pourtant sont indispensables au fonctionnement de la Biosphère dont dépend la survie des sociétés humaines. Certains économistes attendent beaucoup des correctifs du marché. D'autres mécanismes régulateurs existent, mais ils sont généralement associés à une administratition étatique très développée. II reste sans doute beaucoup à inventer.

Cette interrelation entre le développement et l'environnement a tout d'abord été conçue comme une critique du pillage et du gaspillage de notre société de consommation; elle apparaît désormais aussi comme une nouvelle problématique de la pauvreté. Lors de ses travaux préparatoires, la CNUED a établi un lien entre pauvreté et détérioration de l'environnement: l'une aggravant l'autre et réciproquement, créant une spirale catastrophique et pour le milieu naturel et pour les êtres humains. La déforestation, l'érosion des sols ou la sécheresse poussent les populations démunies à surexploiter leurs ressources ou à quitter leur terre et à enclencher un cycle qui, souvent accéléré par la croissance démographique, mènera à la dégradation de l'environnement. A leur tour, la détérioration des ressources naturelles et la baisse conséquente de leur productivité accentuent la pauvreté.

Toutefois un lien existe aussi entre "richesse" et dégradation de l'environnement. Ce qui nous montre que la résolution des problèmes de pauvreté ne mène pas forcément à un développement durable. 


\section{Les voix du Sud}

Parallèlement aux travaux préparatoires de la CNUED, un réseau important reliant plusieurs centaines d'organisations a été mis en place pour faire entendre les voix des organisations et associations non gouvemementales. L'Assemblée générale des Nations Unies a admis que toutes les organisations non gouvernementales (avec ou sans statut consultatif auprès des Nations Unies), celles des milieux économiques, des associations de femmes et de la jeunesse, des syndicats, des populations autochtones s'occupant des questions d'environnement peuvent prendre une part active à la conférence. S'il faut saluer cette ouverture, il faut aussi faire remarquer qu'elle place sur le même plan les représentants des intérêts privés de la grande industrie, les représentants quasi professionnels des grandes organisations anglo-saxonnes de défense de l'environnement et les représentants des associations autochtones qui luttent pour la défense de leur terre. Au surplus, la Conférence de Rio reste formellement intergouvernementale: les organisations non-gouvernementales peuvent tout au plus donner des avis et exercer une pression publique indirecte. En dernière instance seules les délégations gouvernementales participeront directement aux décisions.

D'ailleurs, bon nombre d'organisations du secteur indépendant souhaitent marquer leur autonomie à l'égard des organisateurs officiels de la CNUED. Leurs positions non-officielles exprimeront sans doute davantage la pluralité et la diversité réelles du monde contemporain. Il en fut ainsi à Stockholm en 1972. Toutefois, les intérêts des secteurs indépendants et leurs moyens d'action étant fort différents, cela pourra nuire à la capacité de nombreux groupes à se faire entendre et à exercer leur pression sur la Conférence proprement dite.

En participant au secteur indépendant, de nombreuses organisations et associations des pays du Tiers Monde veulent saisir l'occasion de s'exprimer. En effet, il existe un authentique mouvement, endogène et autonome, pour la défense de l'environnement dans les pays du Sud et leurs représentants estiment, à juste titre, que la conscience écologique ne peut pas et ne doit pas être monopolisée par le Nord (19). Les organisations du Tiers Monde ont aussi l'intention de favoriser la création des réseaux de coopération et l'échange des informations, en marge de la conférence et en la prolongeant. Elles visent également à encourager la participation populaire et l'approfondissement du dialogue sur le terrain (20).

Malgré toutes les mesures prises pour assurer une large et réelle participation des organisations et associations du Tiers Monde aux travaux préparatoires (comme par exemple, la prise en charge des frais de voyage des représentants des pays pauvres), certaines d'entre elles restent sur leurs réserves, quand elles ne sont pas carrément hostiles à l'égard de la manière dont on énonce les problèmes qui seront officiellement discutés à Rio. Après les trois premières sessions du Comité préparatoire, les représentants des pays du Sud déplorent que les problèmes du développement de leurs pays sont escamotés, considérant que les débats portent presqu'exclusivement sur la protection de l'environnement. Certains prétendent qu'on «a noyé le poisson du développement dans une mer d'environnement (21). 
Les clivages politiques et idéologiques qui séparent les pays du Nord et du Sud se retrouvent également dans le secteur indépendant. Entre les ONG du Nord, qui disposent de moyens importants, et celles du Sud, presque complètement démunies, la méfiance s'installe. Ces dernières ont l'impression que ce sont les mouvements écologistes du Nord qui dictent la loi et prescrivent le comportement que les pays du Tiers Monde devraient suivre en matière de la protection de l'environnement. Dès lors, l'intervention des pays du Nord, au nom de la défense de l'environnement, dans les affaires du Sud, est souvent vécu comme une ingérence dans leurs affaires internes. Un rapport publié à New Dehli parle même de “colonialisme environnemental» à propos de la manière dont le World Resources Institute (Washington) évalue la responsabilité des pays du Tiers Monde dans l'émission des gaz à effet de serre (22). Cette immixion est jugée d'autant plus insupportable que l'Occident industrialisé porte une responsabilité historique incontestable et qu'il est fort peu équitable de vouloir imposer aux pays du Tiers Monde des normes écologiques qui ne sont pas - et n'ont pas été - pratiquées au Nord.

Certains pays du Tiers Monde dénoncent les principes de la protection de l'environnement au nom de la défense de leurs intérêts économiques et commerciaux. Ils n'hésitent pas à mettre en accusation les mouvements écologistes du Nord qui, selon eux, véhiculent des idées protectionnistes. Par exemple, les gouvernements des pays tropicaux estiment qu'ils ont le droit d'exploiter leurs forêts pour en tirer du bois qu'ils vendront sur les marchés mondiaux, "pour apporter le développement, l'éducation, les soins de santé et des conditions de vie modernes aux habitants de la jungle." Ils pensent que la campagne contre l'exploitation des forêts est moins fondée sur des considérations écologiques que sur des intérêts commerciaux en vue de développer le marché du bois des pays du Nord (23).

Enfin, beaucoup de chercheurs du Tiers Monde estiment qu'il faudrait dépasser les discours lénifiants des pays industrialisés sur les risques écologiques en montrant concrètement comment on peut gérer l'environnement et en donnant aux pays du Tiers monde des moyens - notamment financiers ou par le biais de transferts technologiques - permettant à ces pays la gestion de leur environnement.

\section{Rio: un enjeu pour les citoyens du monde}

Aujourd'hui "l'environnement" est devenu l'un des enjeux majeurs des négociations internationales au point d'en représenter le troisième pilier avec le commerce et la sécurité. La reconnaissance par la communauté internationale de l'émergence des menaces planétaires, comme la pollution des océans, l'altération de la stabilité des cycles biogéochimiques ou les déréglements du système climatique global, implique pour les Etats un nouveau genre de négociations, déjà souvent dénommée: la diplomatie de l'environnement. C'est ainsi que la Déclaration de La Haye du 11 mars 1989 sur la protection de l'atmosphère affirmait: "comme le problème est planétaire, sa solution ne peut être conçue qu'au niveau mondial." 
Les politiques nationales s'inscrivent dans des contraintes internationales grandissantes et la Conférence de Rio devrait envisager le partage des tâches qui incombent à la communauté internationale afin que celle-ci se rende disponible pour répondre efficacement aux défis de demain. C'est dans la perspective d'une politique internationale de développement durable, écologiquement cohérente, prenant en compte le contexte planétaire de la Biosphère, que se conçoit en effet la signification et l'ampleur sans précédent de l'ambition affichée par cette Conférence des Nations Unies.

L'interdépendance environnement-développement doit définir le niveau minimum de coopération internationale nécessaire pour, d'une part, établir un cadre d'action mondial et, d'autre part, coordonner l'exécution des décisions prisent par la communauté internationale. Une gestion à long terme de l'environnement, ce qui implique l'abolition des activités de production ou de consommation ayant un impact négatif sur l'environnement local et a fortiori global, représente un coût économique (à mettre en balance avec les risques écologiques plus grands encore) et donc une créance sur les ressources d'une collectivité, de l'Etat central voir d'une communauté d'Etats. Dès lors, l'enjeu est triple:

- Comment adapter des politiques environnementales nationales en fonction des atteintes portées au support vital de la société au niveau planétaire, en accord avec la disponibilité des ressources économiques et des choix de politique extérieure?

- Comment abandonner une parcelle de souveraineté régionale ou nationale au profit de la collectivité internationale?

- Comment mesurer les coûts des implications écologiques, économiques et sociales liés à la protection de l'environnement global?

Les Etats-nations actuels, et plus particulièrement ceux "en développement", ne possèdent que rarement la science, la technologie, les ressources humaines et financières, ainsi que les institutions leur permettant de faire face, dans des délais appropriés, non seulement aux bouleversements écologiques à l'intérieur de leurs frontières (aux niveaux local et régional) mais encore aux menaces que représentent les perturbations de l'environnement global. Plus généralement, les responsables politiques se trouvent de plus en plus confrontés à la nécessité de prendre de grandes décisions, engageant l'avenir, sur la base de données scientifiques incertaines, parce que partielles et parfois contradictoires. La complexité des problèmes écologiques, en particulier au niveau global, est telle, l'ignorance sur la géophysiologie de la Biosphère est encore si grande, qu'il s'agit de prendre des décisions, souvent lourdes de conséquences économiques et sociales, sur fond d'incertitude.

En s'efforçant de protéger la couche d'ozone de la stratosphère, de combattre la pollution, de ralentir l'aggravation de l'effet de serre ou de contrôler le mouvement de déchets dangereux, les gouvernements en arrivent, volontairement ou non, à établir de nouveaux obstacles au commerce international et au libéralisme 
économique. La protection de l'environnement dans les pays du Tiers Monde représente des coûts qui ne peuvent être facilement supportés sans une solidarité internationale bien comprise. La répartition sociale du coût de la prise en compte de mesures environnementales dans le cadre du développement d'un pays dépend en partie de la structure économique nationale et internationale. Les pays du Tiers Monde doivent s'assurer l'accès aux ressources nécessaires à leur propre économie, la promotion des exportations tout en protégeant leurs marchés nationaux d'une concurrence extérieure trop puissante. Pour ces pays les mesures écologiques planétaires représentent quelquefois des prétextes destinés, à leurs yeux, à laisser aux pays développés le temps de restructurer leur industrie (cf. le cas des CFC). Cette situation est particulièrement difficile pour l'Afrique dont le commerce ne représente que $4 \%$ environ du commerce mondial.

Le défi est donc bien de convaincre des partenaires sans moyens financiers importants de se consacrer ou de contribuer à la protection de l'environnement planétaire en plus de leurs engagements nationaux dans ce domaine. Si cela se conçoit aisément au niveau local pour améliorer la gestion des ressources dont un bénéfice direct peut être trouvé, comme par exemple dans le cas de la lutte contre la dégradation de la fertilité des sols, c'est beaucoup plus problématique au niveau global. N'oublions pas que les pays du Nord portent une responsabilité nettement plus importante dans les émissions d'éléments perturbants les processus de régulation de la Biosphère, comme les gaz à effet de serre et les CFC. Les exigences de la protection de l'environnement demandent l'élaboration de politiques environnementales nationales capables tout à la fois d'agir dans un espace local et national et d'intervenir au niveau mondial. De même, les politiques mises en place doivent pouvoir comparer ia rentabilité économique des diverses options de protection de l'environnement induites par telle ou telle norme décidée au niveau international et en analyser les retombées sociales. Mais la rentabilité économique ne peut être le seul critère des choix publiques.

La Conférence de Rio devrait pouvoir mobiliser les efforts de la communauté internationale dans la durée. C'est-à-dire, proposer une stratégie mondiale qui ne pénalise ni l'environnement naturel ni le développement humain. Or, le défi est gigantesque, car ce ne sont pas les Nations Unies qui maîtrisent les flux financiers et les macro-processus de l'économie mondiale. Les Nations Unies devraient développer des mécanismes qui permettent à leurs administrations d'élargir les consultations avec les citoyens.

Les synergies entre la gestion de l'espace vital et les processus socio-économiques demandent une certaine harmonie entre les intérêts des Etats, les activités industrielles ou de services des entreprises et les besoins réels des gens. Cette trilogie doit trouver une plate-forme où ces éléments hétérogènes puissent s'exprimer de concert et trouver le meilleur arbitrage possible. La Conférence de Rio pourrait apporter la première pierre à l'édification d'un cadre d'action mondial capable de motiver substantiellement les citoyens du monde entier dans la recherche de solutions à la crise écologique planétaire du développement de l'humanité. 


\section{Rio, une ambition pour l'humanité}

Le développement doit être durable. Pourtant, les mécanismes économiques et financiers internationaux désagrègent les espoirs d'un monde équitable et viable pour tous. Le développement durable sera-t-il celui des privilégiés? La Conférence mondiale de Rio sera-t-elle l'écho d'une impasse ou au contraire le moment historique marquant la rupture avec un ordre économique et politique intemational produisant misère et dégradation de la Biosphère? Cette conférence de 1992 sera-t-elle une nouvelle alliance entre les peuples et la Terre?

C'est là le principal défi: la crédibilité de ce sommet de la planète Terre. Les citoyens du monde doivent être juge et partie; ils doivent scruter la volonté et la sincérité des diplomates, ministres, experts et fonctionnaires qui construisent les institutions du monde de demain. L'enjeu international est de taille. II transcende les Etats-nations, dépasse les capacités novatrices des administrations, bouscule "l'ordre établi" il y a près d'un demi-siècle alors que les super-puissances s'affrontaient dans une rivalité idéologique et politico-militaire qui faillit mener le monde à l'apocalypse de "l'hiver nucléaire". Mais le monde, lui, heureusement, ne s'est pas arrêté: la Biosphère est une source de vie, et donc de durée et de création d'une ingéniosité et d'une imprévisibilité stupéfiantes. La diversité culturelle des peuples et des individus est à l'humanité ce que la diversité biologique est au monde des espèces vivantes. Cette biodiversité et cette ethnodiversité dépassent toutes les frontières politiques et idéologiques. L'interdépendance croissante des diverses sociétés ne doit pas conduire au nivellement mais à l'enrichissement réciproque. Le monde se transforme à une allure vertigineuse, il est en train d'abolir les frontières qui séparaient les hommes et les nations, mais il nous reste à découvrir le nouveau monde de la réconciliation avec la Biosphère, le nouveau "contrat naturel" (Michel Serres).

Les Nations Unies sont issues de la guerre. Elles ont grandi à l'ombre de la terreur de la guerre nucléaire et mûrissent au contact des individus qui se rebellent contre un héritage qui apparaît de plus en plus anachronique. Mais comment, par quels moyens politiques, institutionnels et financiers est-il possible d'inscrire le développement dans le temps long, alors qu'il s'est montré si difficile d'atteindre des résultats satisfaisant dans le temps court?

L'Organisation des Nations Unies, symbole réunificateur de la communauté internationale, doit contribuer à développer les instruments et les mécanismes indispensables pour combler le fossé entre les concepts et le quotidien. Une fois franchi le pas de la signature des différentes conventions qui seront proposées, de la Charte de la Planète Terre, de l'Agenda 21, il restera à mobiliser les ressources humaines et financières, à organiser les structures institutionnelles propres à l'application de ces intentions politiques. Dans le contexte actuel, le risque est grand qu'une fois de plus les actes ne suivent pas les belles paroles.

Mais avant même que puissent se poser des problèmes de mise en oeuvre des résolutions qui seront adoptées, au moment où il convient de finaliser cet article, des tensions sont en train d'émerger. Un peu moins d'une année avant cette conférence des bruits de boycottage se font déjà ententre. 
Trois motifs principaux peuvent être identifiés à ce jour:

- le refus d'ingérence de certains gouvernements dans leur politique en matière d'environnement, réflexe nationaliste allant à l'encontre de la reconnaissance de la dimension supranationale de nombreux phénomènes écologiques,

- le refus d'entrer en matière sur la dette de pays du Nord, en particulier des Etats-Unis d'Amérique. Ce qui peut être considéré plus généralement comme le refus d'aborder globalement les problèmes d'environnement et de développement,

- le refus potentiel de certains gouvernements de prendre en compte les points de vue du secteur indépendant, donc une volonté de s'en tenir à la dimension formellement intergouvernementale de cet évènement en balayant les efforts entrepris, dans le prolongement du travail de la Commission Brundtland, d'ouvrir le débat à l'ensemble des acteurs concernés.

II n'en reste pas moins que la CNUED est en train de faire progresser fortement la prise de conscience international de la dimension écologique du développement. 


\section{Notes :}

1 Développement et Environnement. Rapport présenté par un groupe d'experts convoqué par le Secrétaire Général de la Conférence des Nations Unies sur l'environnement, Founex (Suisse), 4-12 juin 1971, Stockholm, 1971. Pour une vue d'ensemble, qui fait autorité, voir Lynton Keith Caldwell, International Environmental Policy: Emergence and Dimensions, Durham, Duke University Press, 1984; 2 e edition, révisée et mise à jour, 1991.

2 Utilisation et conservation de la biosphère. Actes de la Conférence intergouvernementale d'experts sur les bases scientifiques de l'utilisation rationnelle et de la conservation des ressources de la biosphère, Paris, 4-13 septembre 1968, Paris, Unesco, «Recherches sur les ressources naturelles X», 1970.

3 M. Taghi Farvar et John P. Milton, eds., The Careless Technology: Ecology and International Development, New York, Natural History Press, Doubleday, 1972. (Edition anglaise introduite par Edward Goldsmith, London, Tom Stacey, 1973.)

4 Raymond F. Dasmann, John P. Milton, Peter H. Freeman, Ecological Principles for Economic Development, London, Wiley, 1973.

5 La “conscience écologique», planétaire par définition, implique la redécouverte du concept holistique de la Biosphère. Cette notion du système écologique globale de la Terre remonte aux travaux du savant russe Vladimir Vernadsky, dans les années 1920. Elle a été adoptée par les écologistes les plus scientifiques, mais resta longtemps incomprise du reste de la communauté scientifique et méconnue du grand public, des hommes politiques et des milieux d'affaires. Elle est ravivée de nos jours avec le concept Gaïa de James Lovelock et Lynn Margulis, au coeur du grand débat actuel de la science du "système Terre" (NASA) et du "Global Change" (IGBP/ ICSU). Voir Nicholas Polunin et Jacques Grinevald, “Vernadsky and Biospheral Ecology", Environmental Conservation, 1988, 15(2), pp.117-122.

6 Nicholas Georgescu-Roegen, Demain la décroissance: entropie, écologie, économie, préface et traduction d'I. Rens et J. Grinevald, Lausanne Pierre-Marcel Favre, 1979

7 On trouvera un historique, une bibliographie et un survol de cette problématique dans J. Grinevald, "L'effet de serre de la Biosphère: de la révolution thermo-industrielle à l'écologie globale», Stratégies énergétiques, Biosphère et Société, 1990, 1, pp.9-34.

8 L'Atmosphère en évolution: Implications pour la sécurité du globe, actes de la conférence de Toronto, Canada, 27-30 juin 1988, Environnement Canada, OMM, PNUE, Genève, WMO/OMM - n‥710, 1988. Voir aussi David Adamson, Defending the World: The Politics and Diplomacy of the Environment, London, I.B. Tauris, 1990.

9 Barbara Ward and René Dubos, Nous n'avons qu'une Terre, trad. de l'anglais, Paris, Editions J'ai Lu, 1972.

10 Commission mondiale sur l'environnement et le développement, Notre avenir à tous, Montréal, Les Editions du Fleuve, 1988. Au «développement soutenable» de la traduction française, nous préférons toutefois utiliser ici le concept durable.

11 J.T. Houghton et al., eds., Climate Change: The IPCC Scientific Assessment, Intergovernmental Panel on Climate Change, Cambridge, Cambridge University Press, 1990.

12 C. Mungall et D.J. McLaren, eds., La Terre en péril: métamorphose d'une planète, Société royale du Canada, Ottawa, Les Presses de l'Université d'Ottawa, 1990. Voir aussi Frank Barnaby, ed., The Gaia Peace Atlas, Foreword by Javiez Pérez de Cuéllar, London, Pan Books, 1988.

13 L'expression "secteur indépendant " a été choisie pour englober non seulement les ONG, institutions formellement constituées et reconnues, mais encore tous les grou- 
pes (dont les niveaux d'organisation sont très variables) qui ont en commun leur indépendance vis-à-vis des gouvernements.

14 L'accès à cette information peut se faire à travers les bases de données suivantes: Econet, PeaceNet, HomeNet, ConflictNet, GeoNet, GreenNet, etc. D'autre par le "Centre for our Common Future» publie un mensuel, Network 92. Ce bulletin a pour but de facilirer la transmission des informations sur la Conférence de Rio.

15 Rapport intérimaire sur les institutions, Rapport du Secrétaire général de la Conférence, AVCONF. 151/PC/36, Genève, p.3.

16 Maurice Strong, «Les agents du changement», Sommet Planète Terre Infos, $n^{0} 1$, mars 1991, Genève, Secrétariat de la CNUED, p.1.

17 Cf. Sur la Voie du Brésil. Publication du Centre for Our Common Future, Genève. Notons ici que plusieurs pays du Tiers Monde contestent la dénomination "Charte de la Terren; ils souhaitent de la remplacer par "Déclaration de Rio de Janeiro sur l'environnement et le développement".

$18 \mathrm{M}$. S. Schmidheiny, industriel suisse bien connu, a notamment déclaré à la presse: "Les milieux d'affaires ont leur part de responsabilité dans les problèmes de l'environnement, mais ils sont auss très bien placés pour contribuer à résoudre ceux-ci dans l'avenir."

19 Marc Nerfin, "Environment and development: listen th the south citizen", IFDA Dossier 77, (Nyon), mai-juin 1990, pp.1-2. Bien entendu, dans cette présentation succincte, nous employons les termes "Nord" et "Sud", de même que *Tiers Monden et "pays industrialisés", par pure commodité et faute de place. Inutile de préciser ici que ces termes recouvrent des réalités très diverses, nous en sommes tous bien conscients.

20 Un Comité de coordination internationale (International Facilitating Committee) a été créé sous l'égide du Centre pour Notre avenir à tous en vue de préparer la réunion des organisations non-gouvernementales qui aura lieu à Rio de Janeiro en même temps que la CNUED. Des réunions préparatoires réunissant 200 organisations de 65 pays ont eu lieu à Vancouver, Nyon et Paris. Les participants à ces réunions ont adressé un appel au Comité préparatoire de la CNUED pour lui demander d'attacher une plus grande attention aux aspects “développement" lors de l'établissement de l'ordre du jour.

21 Crosscurrents, Journal ONG indépendant pour la CNUED, n 8, 28-29 août 1991

22 Anil Agarwal et Sunita Narain, Global Warming in an Unequal World: A Case of Environmental Colonialism, New Dehli, Centre for Science and Environment, 1991.

23 Lee Weng Chung, "L'Asie s'oppose aux écologistes occidentaux», Forum du développement, (Genève, Nations Unies), mai-jui 1990. 